\title{
Zweisprachigkeit in den Literaturen Estlands
}

\author{
LIINA LUKAS
}

\begin{abstract}
Bilingualism in the Literatures of Estonia. In a multilingual cultural space such as the (former and contemporary) Baltic region, bilingualism, both oral and written, has been rather normality than exception. This also finds an expression in the literatures of this region. In the following I will examine the phenomenon of bilingualism and multilingualism in the literatures of Estonia in history and today. First, I will examine the historical forms of bilingualism before the foundation of the Republic of Estonia, against the background of the complicated oral and written language relations throughout history. Then I explore their topicality in the interwar and Soviet periods, and today. I also ask about the motivation of the authors to change or mix languages in their work, whether to reach a wider audience or a new poetic quality. Examples are from the work of Paul Fleming, Reiner Brockmann, Jacob Johann Malm, August Kitzberg, Ivar Ivask, Jaan Kaplinski, Igor Kotjuh, Øyvind Rangøy and Veronika Kivisilla.
\end{abstract}

Keywords: bilingualism; exophony; Estonian Literature; Estonian-Russian Literature; Baltic-German Literature

In einem multilingualen Kulturraum wie dem (früheren) Baltikum, ist Zweisprachigkeit, sowohl im mündlichen als auch schriftlichen Gebrauch, eher ein Normal- als Sonderfall gewesen. Auch in den Literaturen dieser Region kommt dies zum Ausdruck. Im Folgenden werde ich dem Phänomen der Zwei- und Anderssprachigkeit in den Literaturen Estlands in der Geschichte und heute nachgehen. Zuerst behandele ich die historischen Formen der Zweisprachigkeit vor der Gründung der Republiken Estland und Lettland, vor dem Hintergrund der komplizierten mündlichen und schriftlichen Sprachverhältnisse von damals. Danach untersuche ich deren Aktualität in der Zwischenkriegs- und Sowjetzeit sowie heute.

\section{Gesprochene und geschriebene Sprachen}

Die Verbreitung der Schrift nach Alt-Livland (dem Gebiet des heutigen Estland und Lettland) fand im Zuge der deutsch-dänischen Eroberung am Ende des 12. Jahrhunderts und zu Beginn des 13. Jahrhunderts ihren Anfang. In Europa 
LUKAS

war das die Periode der „Explosion der Schriftlichkeit“ (Stein 2006: 159-170). $\mathrm{Zu}$ diesem Zeitpunkt hatte die Schrift, die bisher hauptsächlich in der elitären lateinsprachigen Sphäre gepflegt wurde, auch schon die Volkssprachen erreicht und umfasste nun außer der kirchlichen Sphäre auch die säkulare, sie regelte sowohl politische, juristische, administrative als auch gesellschaftliche Beziehungen.

Die Urbevölkerung Alt-Livlands war der Schrift unkundig. Man sprach finno-ugrische und baltische Sprachen: auf dem Gebiet des heutigen Estland die nordestnische Sprache und die südestnische Sprache, auf dem Gebiet des heutigen Lettland Lettgallisch, Semgallisch, Selonisch, Livisch und Kurisch samt deren Dialekten.

Die gesprochenen Sprachen Alt-Livlands hatten ungleichen Zugang zur Schrift. Die Schrift fixierte nicht alle Sprachen, die in dieser Region gesprochen wurden. Die Schrift, die im Zuge der Kolonisation am Ostufer der Ostsee eingeführt wurde, blieb lange Zeit Bestandteil eines kolonialen Gefüges, ein Mittel zur Festigung neuer Machtstrukturen, während die Urbevölkerung mehrheitlich nach wie vor einer mündlichen Kultur zugehörte.

Im Laufe des Mittelalters hat sich in Alt-Livland eine Hierarchie der Sprachen herausgebildet. Auf der obersten Stufe stand das Lateinische, um eine Stufe niedriger folgte die Sprache der Kolonisten: das Niederdeutsche. In den Städten des Baltikums, in denen die schriftliche Verrichtung von Alltagsangelegenheiten schon damals auch volkssprachlich vor sich ging, stammte die Mehrheit der Kolonisten aus Norddeutschland, dem Rheinland und Westfalen (Johansen/Mühlen 1973: 96-97), und bediente sich untereinander des Mittelniederdeutschen - der gemeinsamen Sprache des Hansebundes. Im 16. bis 17. Jahrhundert geriet das Niederdeutsche schon allmählich außer öffentlichen schriftlichen Gebrauch, verdrängt vom aufsteigenden Hochdeutsch. In der mündlichen Sphäre konnte sich das Niederdeutsche immerhin gelegentlich noch bis zum 19. Jahrhundert behaupten. Die geschriebenen und die gesprochenen Sprachen im Baltikum gingen stark auseinander und das gilt auch für das Deutsche: Man sprach im Baltikum eine provinziell gefärbte hochdeutsche Umgangssprache mit zahlreichen niederdeutschen Elementen (Masing 1923), man schrieb aber ein „ordentliches“ Hochdeutsch. Es geht um ein für die Kolonialkultur typisches Phänomen: Einerseits äußerte sich in diesem sprachlichen Gestus ein Abgrenzungsbedarf, ein Versuch, die vor allem durch die Sprache vermittelte kulturelle Identität zu bewahren, andererseits wollte man dadurch die Zugehörigkeit zum „Mutterland“ unterstreichen und sich dort verständlich machen.

Eine kleine russische Minderheit gab es in Alt-Livland schon seit dem frühen Mittelalter. Am Ende des 17. Jahrhunderts dann sind die russischen Altgläubigen 
aus Russland nach Estland eingewandert. Es entwickelte sich eine eigene, archaische russische Mundart, die sie gesprochen haben; gelesen und geschrieben haben sie aber Kirchenslavisch. Obwohl Estland und Livland seit $1710 \mathrm{zu}$ Russland gehörten, spielte die russische Schriftsprache noch im 18. Jahrhundert bei der kulturellen und geistigen Gestaltung der Region noch eine geringe Rolle. Der Verkehr mit der Zentralmacht erfolgte hauptsächlich auf Deutsch. In den Schulen wurde mit Russischunterricht erst am Ende des 18. Jahrhunderts angefangen. Als Kultur- und Bildungssprache im Baltikum behauptete das Russische sich im Laufe des 19. Jahrhunderts, im Zusammenhang mit der (Neu-)gründung der Universität Tartu, zunehmend am Ende des 19. Jahrhunderts unter großem Druck der Zentralmacht (durch die von Alexander III. angestrebte Russifizierungspolitik). Die Zeit der Russifizierung war jedoch zu knapp, um die bisherige kulturelle Dominante ersetzen zu können. Als Zeichen des zunehmenden russischen Einflusses finden sich aber im Deutschbaltischen zahlreiche Lehnworte aus dem Russischen.

Auf der untersten Stufe der Sprachhierarchie des Baltikums standen die Sprachen der Urbevölkerung. Dies waren Sprachen, die lange Zeit nicht schriftlich verwendet wurden. Semgallisch, Selonisch und Kurisch waren schon im 16. Jahrhundert ausgestorben. $\mathrm{Zu}$ einer Schriftsprache brachten es nur die übrigen vier Sprachen: das Südestnische, das Nordestnische, das Lettische und das Lettgallische. Die neuen Schriftsprachen waren von örtlichen Pastoren, die in der Regel deutsche Muttersprachler waren, geschaffen worden, um die christlichen Glaubensinhalte auch in den einheimischen Sprachen des Baltikums besser vermitteln zu können. Im Laufe des 17. Jahrhunderts fing man allerdings an, in diesen Sprachen auch immer mehr säkulare Texte juristischen, wirtschaftlichen, belletristischen und sonstigen Inhalts zu verfassen.

Da die Sprachkenntnisse der "Schöpfer" der estnischen und lettischen Schriftsprachen nicht gut waren und es weder Wörterbücher noch Grammatiken gab (die mussten erst zusammengestellt werden), wurden die in dieser Weise entstandenen Schriftsprachen von den mündlichen Trägern dieser Sprachen als fremd empfunden und nur allmählich übernommen. Das Schriftestnische (bzw. -lettische) fungierte als Instrument der Christianisierung und Kolonialisierung. Die Diskrepanz zwischen Mündlichkeit und Schriftlichkeit ist im Baltikum wohl um einiges langfristiger und prägender gewesen als es in vielen anderen Regionen Europas der Fall war. Erst im Laufe des 19. Jahrhunderts erweiterte sich allmählich das Verwaltungsgebiet der Schrift und aus einem überaus elitären, exklusiven Medium zum Wissenserwerb wurde ein Medium für jedermann, vom Mittel der Fremdbeschreibung eines der Selbstbeschreibung. Das Baltikum ist ein sehr interessanter Fall für die Erforschung dieser (Aneignungs-, Übersetzungs-, Adaptions-)Verhältnisse. Dennoch unterschied sich die Situation 
LUKAS

erheblich von späteren „Kolonialsituationen“, bei denen eine kolonisierende Kultur den Kolonisierten ihre Sprache aufgezwungen hat. Im Baltikum hat die kolonisierende Kultur vielmehr die Sprache der Kolonisierten erlernt und versucht, sie nach eigenen Modellen, nach bekannten Mustern aus dem Deutschen oder Lateinischen, zu gestalten.

Die neuen Schriftsprachen, die sich auf diese Weise herausbildeten, übten ihren Einfluss auch auf den mündlichen Gebrauch dieser Sprachen aus, der Wortschatz der gesprochenen Sprachen veränderte sich, neue Begriffe wurden geprägt, alte Begriffe gerieten infolgedessen außer Gebrauch, grammatikalische und syntaktische Konstruktionen änderten sich.

Während im schriftlichen Gebrauch eine deutlichere Hierarchie der Sprachen existierte, war im mündlichen Gebrauch der Sprachaustausch lebhafter, Übergänge waren fließender, je nach Verwendungssituation und der sozialen Position der Sprecher, bis zu den Zwischen- oder Übergangsformen wie Deutschbaltisch, Kleindeutsch, Halbdeutsch bzw. Wachholderdeutsch, wie man das fehlerhafte Deutsch der sich assimilierenden Esten nannte. Rund 25\% der estnischen Wortstämme sind Entlehnungen aus dem Niederdeutschen. Der Einfluss des Hochdeutschen (zugleich der späteren deutschbaltischen Sprache/ Mundart) auf die Sprachen der Esten und Letten ist viel geringer. Nicht selten wurde das eine oder andere Wort aus dem Niederdeutschen auf dem Umweg über das Estnische oder das Lettische sogar ins Deutschbaltische zurückentlehnt. Auch in das deutschbaltische Sprachgut sind viele Lehnwörter aus dem Estnischen bzw. Lettischen, aber auch aus dem Russischen eingeflossen.

Dass viele deutschbaltische Kinder adligen Geblüts unter der Obhut estnischer und lettischer Ammen und Dienstleute das Estnische bzw. Lettische als Erstsprache erwarben, ist ein in der deutschbaltischen Literatur verbreitetes Motiv. Unvermeidlich war die Zweisprachigkeit sicherlich für die baltischen Literaten (wie Bildungsbürger im lokalen Sprachgebrauch üblicherweise hießen), deren begehrtestes Ziel es war, eine Pastorenstelle zu bekommen, die man ohne Kenntnis der lokalen Sprachen nicht ausüben konnte (noch durfte). Andererseits war das Deutsche auch für die Akademiker estnischer bzw. lettischer Herkunft die Bildungssprache schlechthin, so dass gebildetere bzw. sozial aufgestiegene Esten und Letten auch zu Hause meistens zum Deutschen wechselten. Noch zu Beginn des 20. Jahrhunderts bediente man sich in gebildeten Kreisen oft des Deutsch(baltisch)en. Im Jahre 1891 schrieb Oskar Kallas, ein namhafter Folklorist, später auch Journalist und Botschafter der Republik Estland in London, in seinem (auf Deutsch verfassten) Tagebuch, dass er keine estnische Familie kenne, in der man unter sich Estnisch spreche. Die bekannteste estnische Lyrikerin Marie Under machte ihre ersten Schritte auf ihrem 
Dichterweg auf Deutsch und fing erst später, auf Anraten ihres Freundes, des Künstlers Ants Laikmaa, an, auf Estnisch zu dichten.

\section{Zweisprachigkeit in der Literatur}

Baltische Literaten waren bis zum Ende des 19. Jahrhunderts fast immer mindestens zweisprachig, ungeachtet ihrer nationalen oder ethnischen Herkunft. Ihre Bildungssprache war in der Regel Deutsch, außerdem verstanden bzw. sprachen sie, lebten sie im nördlichen Teil Livlands oder in Estland, meistens mehr oder weniger gut Süd- oder Nordestnisch, im lettischsprachigen Teil des Baltikums Lettisch oder Lettgallisch, mitunter konnten sie sich auch einer dieser Sprachen schriftlich bedienen. Ihr lokales, tägliches Deutsch stand unter dem Einfluss dieser Sprachen bzw. deren Mundarten. Mehrsprachigkeit, die Fähigkeit, sich sowohl schriftlich als auch mündlich verschiedener Sprachen zu bedienen, war ein wichtiger Charakterzug dieses Kulturraumes. Selbstverständlich kommt dies auch in der Literatur zum Ausdruck.

Im Folgenden werde ich einige Beispiele für den Gebrauch der Zweisprachigkeit in der Literatur des Baltikums anführen. Ich gehe dabei von der von Jaan Undusk im Jahre 1992 entworfenen, immer noch durchaus aktuellen Typologie des estnisch-deutschen Literaturtransfers (Undusk 1992) aus, die zwischen Formen verschiedenster Kontakte literarischer Art unterscheidet. Mich interessieren an dieser Stelle jedoch lediglich die Kontakte, die sich mit dem Phänomen der Zweisprachigkeit in Verbindung bringen lassen. Es lässt sich zwischen einer sprachinternen, textinternen und autorinternen Zweisprachigkeit unterscheiden.

2.1 Mit sprachinterner Zweisprachigkeit meine ich Texte, die in einer Mischsprache entstanden sind. Der deutschbaltische Dialekt ist selbst schon gewissermaßen eine solche Mischsprache, in die estnische bzw. lettische, russische oder französische Wörter eingebettet sind.

Ein frühes Beispiel ist ein kleines Fragment aus dem Gedicht „Lieffländische Schneegräfin“ (Fleming 1638) von Paul Fleming (1609-1640), in dem Hochdeutsch, Niederdeutsch und Estnisch sich mischen:

Die Braut / bald rot / bald blaß, fing endlich an zu reden: „Wat schal ich arme Kind? Gott weht, wat sy my theden!“

Das ander /Ycks / Kacks / Koll ${ }^{1}$ hub sie auff Undeutsch an,

Das ich noch nicht versteh', und auch kein Gott nicht kan.

1 Eins, zwei, drei auf Estnisch. 
LUKAS

Literarische Texte im deutschbaltischen Dialekt wurden jedoch selten verfasst, in der Regel geschah dies nur in ,niederen' Literaturgattungen, wobei das Deutschbaltische die Funktion von Parodie, Ironie oder Witz innehatte. Das Deutschbaltische (mit all seinen Jargons) wurde dem Bereich des Komischen zugeordnet und kam am systematischsten zum Einsatz in der sogenannten halbdeutschsprachigen Dichtung — in der makkaronischen Dichtung $^{2}$ des Baltikums vor allem des 19. Jahrhunderts. In dieser Gattung werden zwei Sprachen zur Erzielung eines komischen oder parodistischen Effektes vermischt, indem Morphologie und Syntax der deutschen Sprache auf den Wortschatz des Estnischen bzw. des Lettischen übertragen werden, wobei die Phonologie dem Estnischen angepasst wird, z.B. werden die stimmhaften Konsonanten durch die stimmlosen und Doppelkonsonanten durch einen Konsonant ersetzt, das h weggelassen usw. (Über die Merkmale des Halbdeutschen siehe Ariste 1981).

Die Oberpahlsche Freundschaft (1818/1857) des Tallinners Jacob Johann Malm (1796-1762) ist das erste und bekannteste, geradezu wegweisende Gedicht dieser Gattung gewesen (siehe dazu Lehiste 1965).

\author{
Vart', tenkt' ich mal in meine Sinn, \\ Willst wahren toch heinmal \\ Su Wreind nach Oberpalen in! \\ Und ging nu in tas Tall ${ }^{3}$ \\ Und nehmt tas Wuchs mit lange Wanz \\ Und pannt tas wor tas $\mathrm{Saan}^{4}$; \\ Tann nehmt' ich meine Mütz und Ans \\ Und wangt' su jagen an; \\ Und nu katsait turch Tuchk und Tolm ${ }^{\text {s }}$ \\ Ich tuhhat neljad ${ }^{6}$ wort. \\ Und wie tas Wind war üks, kaks, kolm ${ }^{7}$ \\ Ich an tas Tell und Ort.
}

\footnotetext{
2 Den Begriff der Makkaronie im baltischen Kontext hat zuerst Maie Kalda eingeführt (Kalda 2000).

3 Estn. Stall

4 Estn. Schlitten

5 Estn. Asche und Staub

6 Estn. mit grosser Schnelligkeit

7 Estn. eins, zwei, drei
} 


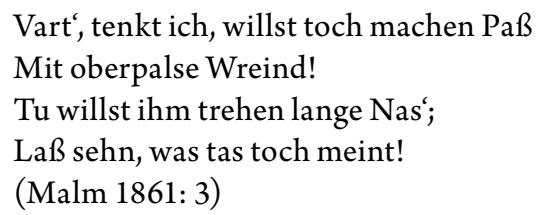

Auch in der estnischen Literatur ist dieser Typus bekannt. Ein Dialog aus der Erzählung Veli Henn (1901) von August Kitzberg (1863-1955, Kitzberg 2002: 271) klingt wie folgt:

Kniks-Mariihen: „Bitte,“ ütles Mariihen. „Astuge aita, sääl on toolisid, ja võite ennast natuke erhoolida.“

„Herr Lehepuu, üks väga peenike kawalier, - herr Birkenbaum, minu Freundini Bräutigam, - herr Sissa, minu Tänzer, kui Vereinis ball oli, - herr Enilane, ka üks hää Tänzer...

Anders als bei Malm ist hier die syntaktische und grammatikalische Basissprache Estnisch, in das deutsche Wörter oder Ausdrücke eingebettet und grammatisch angepasst sind. Jedes zweite Wort in der Rede von KniksMariihen ist deutsch: Bitte, erhoolida (erholen), Herr, Kawalier, Freundin, Bräutigam, Tänzer, Verein, Ball.

Ungeachtet der Basissprache wird in dieser Dichtung ein bestimmter sozialer Typus dargestellt, ein ,Emporkömmling' meistens estnischer bzw. lettischer Abstammung oder aber auch ein sozial heruntergekommener Deutscher (ein sogenannter Klein-Deutscher), der seine Identität aufgegeben hat bzw. seine Position in der Gesellschaft ändern möchte und seine (vermeintliche) Bildung gern hervorkehrt. Dabei kann er z.B. ,gehobene' deutsch- oder französischsprachige Sätze verwenden. Vahur Aabrams (Aabrams 2007) hat die halbdeutschsprachige Dichtung als Erscheinung einer karnevalesken Kultur im Sinne von Michail Bachtin interpretiert.

2.2 Unter textinterner Zweisprachigkeit verstehe ich die Verwendung zweier Sprachen in einem Text, ohne Morphologie und Syntax einer Sprache an die Ziel- oder Basissprache anzupassen. Abrupt wird von einer Sprache in die andere übergegangen, wobei die Sprachen eine bestimmte kulturelle Funktion im Text haben. Diese Art von Mehrsprachigkeit nahm ihren Anfang mit kirchlichen Texten im 16. Jahrhundert und war noch in der zweiten Hälfte des 19. Jahrhunderts im Baltikum gebräuchlich. Ein früheres, charakteristisches Beispiel sind die hauptsächlich estnischsprachigen Predigten (1600-1608) des Tallinner Pastors Georg Müller (1570-1608), in denen deutsche und lateinische Passagen verwendet werden. Aus späterer Zeit könnte man das Stück Die 
LUKAS

väterliche Erwartung, eine ländliche Familien Scene in Esthland, mit Untermischten Gesängen (1789) von August von Kotzebue (1761-1819) hervorheben, das auf der Bühne des Revaler Liebhabertheaters uraufgeführt wurde. Der dritte Akt des Stückes beginnt mit einem estnischsprachigen Dialog und die ganze Parallelhandlung findet auf Estnisch statt.

Textbeispiele für texinternen Bilinguismus aus dem 19. Jahrhundert sind etwa der zweisprachige Briefwechsel von Lydia Koidula (1843-1886), der ersten estnischen Lyrikerin, und Friedrich Reinhold Kreutzwald (1817-1903), dem Verfasser des estnischen Nationalepos Kalevipoeg (Kalews Sohn).

2.3 Mit autorinterner Zweisprachigkeit ist der Fall gemeint, bei dem ein Autor sich zweier Sprachen bedient, indem er seine Schriftsprache entsprechend der Funktion, Gattung, dem Stil und Adressaten des Textes wählt, aber in einem Text durchgehend eine Sprache verwendet. Dies ist die Situation, die in der heutigen Literaturwissenschaft als Anderssprachigkeit bzw. Exophonie bezeichnet wird (Siehe Arndt et al 2007). Allerdings konnte im historischen Baltikum ein Autor auch in mehreren Sprachen schreiben wie das etwa bei der Gelegenheitsdichtung des 17. Jahrhunderts nicht selten der Fall war. Das allererste estnischsprachige Gedicht wurde im Jahre 1637 von dem gebürtigen Mecklenburger Reiner Brockmann (1609-1647), ein Professor des Revaler Gymnasiums, verfasst und trug den lateinsprachigen Titel Carmen alexandrinum esthonicum ad leges Opitij poeticas compositum. Wie in der Barockdichtung üblich, hat Brockmann auf Latein, auf Griechisch, auf Deutsch und nun auch auf Estnisch Gedichte verfasst. Sein Plädoyer für die estnische Sprache auf Deutsch klingt wie folgt:

Andre mögn ein anders treiben;

Ich hab wollen Esthnisch schreiben.

Esthnisch redet man im Lande /

Esthnisch redet man am Strande

Esthnisch redt man in der Mauren

Esthnisch redden auch die Bauren

Esthnisch redden Edelleute

Die Gelährten gleichfalls heute

Esthnisch redden auch die Damen

Esthnisch, die aus Teutschland kamen,

Esthnisch reden jung' und alte.

Sieh, was man von Esthnisch halte?

Esthnisch man in Kirchen höret

Da Gott selber Esthnisch lehret.

Auch die klugen Pierinnen 
Jetzt das Esthnisch lieb gewinnen.

Ich hab wollen Esthnisch schreiben

Andre mögn ein andres treiben.

(Brockmann 2000: 94-95)

Die Landprediger Gotthard Friedrich Stender (1714-1796) und Johann Wilhelm Ludwig Luce (1756-1842) haben ihre aufklärerischen Schriften auf Deutsch, ihre volksaufklärerischen Werke auf Lettisch bzw. Estnisch verfasst; der Dichter Kristian Jaak Peterson (1801-1822) hat seine Arbeiten zur Sprache und Religion auf Deutsch geschrieben, sein dichterisches Werk ist aber in estnischer Sprache verfasst (mit Ausnahme von einigen deutschsprachigen Gedichten). Der Este (bzw. der estnischstämmige) Friedrich Robert Faehlmann (1798-1850) verfasste seine berühmten Estnischen Sagen auf Deutsch, der Deutsche (bzw. der deutschstämmige) Georg Julius von SchultzBertram (1808-1875) wiederum sein Epos Ilmatar (1870) auf Estnisch. Das estnische Nationalepos Kalevipoeg (unter dem Titel: Kalewipoeg. Eine estnische Sage, 1857-1861) erschien zum ersten Mal parallel auf Estnisch und Deutsch. Die Wahl der Schriftsprache war meistens abhängig von der angestrebten Funktion des Textes und von dessen Adressaten (Undusk 1999). Auch in gelehrten Kreisen des beginnenden 20. Jahrhunderts kam es noch vor, dass man die Sprache nach der Funktion und Gattung des Textes und nach dem Adressaten wechselte. Zum Beispiel dichtete Axel Kallas (1890-1922) sowohl auf Deutsch als auch auf Estnisch. Im Jahre 1912 veröffentlichte er seinen deutschsprachigen Lyrikband Am Moor (Dorpat 1912); im Jahre 1920 erschien noch ein deutschsprachiger "futuro-kubistischer" Band Nervenvibrierungen im Tintengewande: Futuro-kubistsches. Ein Jahr darauf wechselte er seine Dichtungssprache und gab zwei Gedichtbände auf Estnisch heraus.

\section{Postkoloniale Zweisprachigkeit in Estland}

Erst durch die Geburt der neuen selbständigen Staaten Estland und Lettland im Jahre 1918 haben Estnisch und Lettisch den Status einer Staatssprache erreicht. Damit einher ging auch die Möglichkeit, die Sprache in allen Lebensbereichen zu verwenden, auf dem Gebiet der Wissenschaft etwa bedeutete das großteils das Betreten von Neuland.

In Estland wurde vom neuen Staat allen nationalen Minderheiten (8\% Russen und 1,7 \% Deutsche, Schweden, Ingermanländer und Woten) eine damals weltweit beispiellose Kulturautonomie gewährt und auch die staatliche Kulturförderung war nicht explizit auf die Staatssprache beschränkt (Hasselblatt 1997: 37-46). Obwohl die deutsche Bevölkerungsgruppe Estlands 
LUKAS

immer kleiner wurde (im Jahre 1881 machte sie 5,3\%, im Jahre 1887 3,5\%, im Jahre 1922 1,7 und im Jahre 1934 nur noch 1,5\% von der gesamten Bevölkerung aus), spielte sie immer eine große Rolle auf politischem, wirtschaftlichem und kulturellem Gebiet. Neben estnischsprachigen Schulen existierten in Estland während der Zwischenkriegszeit auch deutschsprachige und russischsprachige, es erschienen Zeitungen und sonstige Periodika in einer Reihe von Sprachen (Deutsch, Russisch, Jiddisch, Schwedisch). Im Universitätsunterricht wurde in den 1920er Jahren noch Deutsch und Russisch verwendet (estnischsprachige Fachliteratur gab es zunächst noch kaum), aber der Übergang zum Estnischen vollzog sich konsequent und letztlich auch erfolgreich.

Obwohl zur Zeit der Republik Estlands die Intellektuellen und viele Staatsbürger und Einwohner des Landes faktisch mehrsprachig waren, imstande, sich auf Estnisch, Deutsch und Russisch, in „den drei lokalen Sprachen“, wie es hieß, auszudrücken, nahm in der Literatur Mehrsprachigkeit allmählich ab.

Nach dem Zweiten Weltkrieg zerfiel die estnische Literatur in zwei Teile, und da das Schicksal viele SchriftstellerInnen als Exilierte ins Ausland - nach Schweden, Kanada, Australien, in die Vereinigten Staaten oder sonstwohin - verschlagen hatte, wurde Exophonie in der estnischen Literatur zu einem Phänomen, das vor allem für die Textproduktion von ExilautorInnen charakteristisch war. Während die erste Generation estnischer ExilautorInnen vorwiegend auf Estnisch geschrieben hat, konnten die im Kindesalter aus dem estnischen Sprachraum Herausgerissenen gelegentlich bereits in zwei Sprachen schreiben. Elin Toona (1937) hat ihren im schwedischen Lund 1969 erschienenen Roman Lotukata auf Estnisch und Englisch (unter dem Titel In Search of Coffee Mountains) verfasst. Karin Saarsen (1926-2018) hat auf Schwedisch und Estnisch geschrieben. Ihr letzter Lyrikband Lõvi ja orhidee (Löwe und Orchidee) enthält Gedichte auf Estnisch, Schwedisch, Englisch, Deutsch und Französisch.

Auf Deutsch haben die estnischen ExilautorInnen allerdings nur noch selten geschrieben. Fast eine Ausnahme bildet das Werk von Ivar Ivask (19271992). Ivask wurde 1927 in Riga (man könnte sagen: auf der größten deutschen Sprachinsel des Baltikums) als Sohn eines estnischen Vaters und einer lettischen Mutter geboren und wuchs mehrsprachig auf: neben Estnisch wurde zu Hause auch Lettisch und Deutsch gesprochen. Unter dem Einfluss von Rainer Maria Rilke schrieb der 16-jährige seine ersten Gedichte in deutscher Sprache. Vor der sowjetischen Besatzung floh er 1944 nach Deutschland, erhielt seine akademische Bildung als Germanist in Marburg, wurde später Professor für vergleichende Literaturwissenschaft an der University of Oklahoma und Chefredakteur der angesehenen Fachzeitschrift World Literature Today. Auf seine Initiative geht auch der renommierte Neustadt International Prize 
for Literature zurück. Ivask schrieb seine Lyrik auf Estnisch, Englisch und Deutsch (die nichtestnischen Werke sind Gespiegelte Erde (1967) und Baltic elegies (1987)).

Im Vorwort zu Ivasks deutschsprachigem Gedichtband schreibt Herbert Eisenreich, dass Ivar Ivask mit seinem Gedichtband der deutschsprachigen Lyrik etwas Neues brachte und erklärt das wie folgt: Ivasks deutsche Gedichte gewinnen, sagt er,

ihre Eigentümlichkeit und ihren doppelten Wert, den des Gefühlten und den des Gesagten, offensichtlich in dem Prozess und durch den Prozess der Aneignung: $\mathrm{da}$, in dem doch fremden Idiom, wurde einem nichts geschenkt, man musste es teuer erkaufen, verzweifelt erobern - und gelangte just dadurch weit über das Konventionelle hinaus (mit den geradezu unverfroren zusammengesetzten Hauptwörtern, zum Beispiel, oder in der naiven Wiedergewinnung der Anschaulichkeit von scheinbar verbrauchten Wort-Bildern). Oder anders ausgedrückt: Ich merke, dass da jemand Fremder meine Muttersprache spricht, und in dieser winzigen Fremdheit klingt sie, im allergenauesten Wortsinn, unerhört, in dieser winzigen Fremdheit hat sie nun wieder jungfräuliche Reinheit. Und mit dem fremden Akzent sagt sie, diese Sprache, mir mehr, als sie`s in meinem eignen, gleichsam routinierten Gebrauch vermag. [...] In diesen Versen [ist] fortgebildet, wie man im andern, im Fremden, zu sich kommt. Und vice versa.

(Eisenreich 1967: 7)

Die im Exil geborene Generation schrieb allerdings (fast) nicht mehr auf Estnisch, ihre Schaffenssprache wurde meistens die Sprache ihrer jeweiligen Wahlheimat.

In Sowjet-Estland gelang es gleichzeitig, allen Russifizierungsversuchen zum Trotz, die estnischsprachige Bildung auf allen Ebenen - eine Errungenschaft der Vorkriegszeit - aufrechtzuerhalten, auch wenn gelegentlich die Verwendung russischer Sprache von den Autoritäten stark bevorzugt und gefördert wurden. Der westliche Teil der Sowjetunion ist ein aufschlussreiches Untersuchungsfeld für all diejenigen, die sich für die Frage interessieren, wie Sprachen auf historisch-politische Umstände reagieren und ihrerseits darauf Einfluss nehmen können. Im Fall Sowjet-Estlands folgte auf den politisch und bildungspolitisch mit allen Mitteln geförderten Sprachwechselkurs zum Russischen hin als Reaktion eine verstärkte Pflege der estnischen Sprache, bis hin zur Konservierung, eine von der Mehrheit der Urbevölkerung wohl als Selbstverständlichkeit empfundene Verwendung der estnischen Sprache in nahezu allen gesellschaftlichen Bereichen. Man erwarb bis zum Ende der Sowjetzeit zwar relativ gute Kenntnisse der russischen Sprache, aber die 
LUKAS

Übernahme von Sprachelementen aus dem Russischen blieb trotzdem eher recht gering und oberflächlich.

Estnisch-russische Zweisprachigkeit kam und kommt im Alltag zwar vor (siehe Verschik 2005; Verschik-Bone 2018), in der Literatur gibt es sie trotzdem nur vereinzelt.

\section{Exophonie in der/den heutigen Literatur(en) Estlands}

Von den Fällen der Exophonie in der zeitgenössischen estnischen Literatur hängt keiner mit der deutschen Sprache zusammen. Paradoxerweise hat das Ereignis, das man gemeinhin mit der Absicht Deutschlands, Osteuropa sowohl politisch als auch kulturell und sprachlich zu unterwerfen, assoziiert, im Baltikum das Gegenteil erreicht. Die Umsiedlung (fast) aller Deutschbalten 1939 (und 1941) aus dem Baltikum „heim ins Reich“, hat mehr oder weniger abrupt und wohl auch unwiederbringlich die estnisch/lettischdeutsche Zweisprachigkeit beendet. Jenes Ereignis hat innerhalb kürzester Zeit die deutsche Sprache ihres bisherigen kaum umstrittenen Status als erster Fremdsprache und erster Wissenschaftssprache der baltischen Region beraubt.

Deutsch wird in den Literaturen des Baltikums heutzutage nicht mehr verwendet (allenfalls als Stilmittel (Lokalkolorit) in historischen Romanen und Erzählungen, z.B. von Jaan Kross (1920-2007); oder Mati Unt (1944-2005) in seinem estnischsprachigen Brecht-Roman, der den deutschsprachigen Titel hat: Brecht bricht ein in der Nacht (1997).

Im heutigen Estland kann es in folgenden Fällen zu Zweisprachigkeit kommen: Die üblichen Sprachpaare sind Estnisch und Englisch, Estnisch und Russisch, Estnische Standardsprache und lokale Mundarten (Setukesisch, Werro-, Mulgi-, Kihnuestnisch usw.).

In welcher Form (textintern, autorintern, sprachintern) äußert sich Zweisprachigkeit in der zeitgenössischen Literatur Estlands?

\subsection{Sprachinterne Zweisprachigkeit}

Obwohl der sprachinterne Bilingualismus inzwischen aufs Neue ein alltägliches Phänomen geworden ist (in der estnischen Umgangssprache vor allem jüngerer Leute wimmelt es von Elementen aus dem Englischen, die Umgangssprache der Russen Estlands kommt aber darüber hinaus, so scheint es, nicht mehr ohne estnische Worte aus), trifft man diese Art von Zweisprachigkeit in der Literatur eher selten an. Als ein Beispiel können die Gedichte von Veronika Kivisilla angeführt werden (Kivisilla 2018: 5.): 
Zweisprachigkeit in den Literaturen Estlands

\section{JUUNI 2018}

Tänahommikune vestlus kassapidaja Svetlanaga Pääsküla poes. Olime ühel meelel:

Tema: „Oi, kakaja ilm, tolko päike i päike!“

Mina: „Jah, milline pagooda, ainult solntse ja solntse! Aga otšen dožd vaja!“ Tema: „Da, väga vihm naada!“

Der im Text angeführte Dialog ist in der estnisch-russischen Mischsprache, die russischen Wörter sind in estnischer Schrift weitergegeben und so ist der Text nur den Estlands-Russen und den Esten, die etwas Russisch können, verständlich. Die den Alltag poetisierenden Texte von Kivisilla schöpfen aus der engsten Umgebung; die Verkäuferin Svetlana, die diese Sprache verwendet, ist in ihnen eine wiederkehrende Figur.

\subsection{Textinterne Zweisprachigkeit}

Auch textinterne Zweisprachigkeit ist eine seltene Erscheinung und lässt sich an unerwarteten Orten entdecken, z.B. in der Lyrik von Øyvind Rangøy, eines Norwegers, der in Estland seine Wahlheimat gefunden und angefangen hat, auf Estnisch zu dichten. Sein Gedicht „Kodeveksling. Koodivahetus“ (Rangøy 2019) ist zweisprachig:

Eg er norsk. I det er eg heime, slik ein er heime som legg frå seg Sekken. Ser at bislaget er der som før, med lukter av barndom. At kjeledressen er min no, for nokon er død. Olen eesti keeles ka olemas.

Kui õunapuule poogitud pihlakaoks. Kunagine teine puu on Praegu minugi väikeste õunte mahlas. Lugu, mis kunagi kaugel Mere ja okastraadi taga vaid virvendas, on nüüd ka minu. [---]

Auch die schon erwähnte Dichterin Veronika Kivisilla kann gelegentlich diese Art von Zweisprachigkeit verwenden, indem sie Umgangsgespräche (auf Englisch) in ihre Texte einbettet (Kivisilla 2018: 25).

\subsection{Autorinterne Zweisprachigkeit (Exophonie)}

Am häufigsten kommt die (autorinterne) Zweisprachigkeit - die Exophonie in der Literatur Estlands vor und es lassen sich folgende Fälle unterscheiden:

1. Exophonie zwischen Estnisch und Englisch (ein estnischer Autor fängt auf Englisch zu schreiben an). Das hat weniger mit der persönlichen Identität bzw. einem sprachlichen Hintergrund des Autors zu tun, vielmehr ist hier wohl von einem Versuch auszugehen, auf diese Art und Weise ein breiteres, 
LUKAS

internationales Publikum zu erreichen. Unter Liederautoren in der estnischen Popmusik ist das seit den 1990er Jahren schon fast ein Normalfall.

2. Russisch-estnische Exophonie (siehe auch Belobrovtseva 2018) wird vertreten von estnisch-russischen AutorInnen, die gelegentlich auf Estnisch schreiben. Das kommt immer noch eher selten vor. Die Literatur der Russen Estlands ist meistens in russischer Sprache geschrieben. Eine Ausnahme, die die Regel bestätigt, wäre Igor Kotjuh, der seine Texte vorwiegend auf Russisch schreibt, im Jahre 2007 aber auch einen estnischsprachigen Gedichtband Teises keeles (In einer anderen/zweiten Sprache) herausgebracht hat.

In einer anderen Sprache

1.

Es gibt die Muttersprache und eine andere Sprache.

Aber der Mensch ist derselbe.

2.

Es gibt das Leben und die Dichtung.

Jeder Dichter ist ein Mensch, nicht andersherum.

3.

Man kann eine andere

Sprache beherrschen

im Leben oder in der Dichtung, von Geburt an

oder danach.

Dennoch wird diese Sprache für ihn eine ewig andere bleiben. (Kotjuh 2007: 31-33)

Versuch einer Selbstbestimmung

Sich den Esten zurechnen - mit Russisch als Muttersprache.

Sich den Russen zurechnen - das Temperament stimmt nicht.

Sich einen Europäer nennen - ein Vorrecht Auserwählter.

Oder einen Weltbürger — zu abstrakt. 
Man muss schlicht und einfach ein Mensch sein.

Wird das wohl verstanden?

(Kotjuh 2007: $30^{8}$ )

Seltener gibt es AutorInnen mit estnischen Wurzeln, die angefangen haben, auf Russisch zu schreiben. Einer von ihnen ist Priit Parmakson, dessen literarisches Werk im Grenzgebiet von Sprachen (Russisch, Estnisch, Englisch) und Kunstsprachen (Literatur, visuelle Künste, z.B. Graphik) entsteht. Seine Muttersprache ist Estnisch, aber auf Russisch zu schreiben hat er angefangen als ideologische Geste nach den Ereignissen um den Bronzenen Soldaten (das umstrittene Denkmal für die im Zweiten Weltkrieg gefallenen Soldaten der Roten Armee in Tallinn, das von der estnischen Seite als Symbol der Annektierung Estlands, von der russischen Seite als das Symbol der Befreiung angesehen wird) im Jahre 2007. Die Motivation des estnischen Romanschriftstellers Kalle Käsper, seine dichterische Sprache zu wechseln, war von anderer Art: Sein russischsprachiger Roman $\Psi y \partial o$ (2018) entstand als Abschiedsroman von seiner todkranken, russischsprachigen Frau.

3. Drittens kann das Phänomen der Exophonie im Zusammenhang mit der sogenannten Mundartenliteratur (vor allem auf Werroestnisch, Setukesisch) behandelt werden. Estnisch ist für die meisten estnischen SchriftstellerInnen heutzutage zwar die erste Schriftsprache, eine Mundart hingegen (bzw. eine lokale Sprache) lernt man in der Funktion einer Schriftsprache erst nach dem Erwerb der normierten estnischen Sprache kennen und danach gegebenenfalls auch als Literatursprache zu verwenden, da die einheitliche Schulbildung in Estland (seit etwa 100 Jahren überall) nur auf Schriftestnisch stattfindet. Die Zahl der AutorInnen, die nur auf Werroestnisch und Setukesisch und nicht in estnischer Standardsprache schreiben, ist heute dennoch relativ groß (Kauksi Ülle, Merca, Andreas Kalkun u.a.).

4. Dass ein Nichtmuttersprachler sich des Estnischen als dichterischer Sprache bedient, kommt selten vor. Eine Ausnahme ist die Lyrik des schon erwähnten Øyvind Rangøy, in der er auch seine exophone Situation reflektiert:

Außerhalb der Sprache

Wurzeln schlagen in einer anderen Sprache ist ein In-die-Ehe-Treten in umgekehrter Richtung: man wird zwei Fleisch, zwei Seelen. Der Gang der Dinge ist unter Umständen schmerzhaft, wunderbar und unvermeidlich. Innerlich sich zweizuteilen ist ein erstaunlich schneller Prozess.

8 Deutsche Übersetzung von Vahur Aabrams. 
Wer bin ich außerhalb der Sprache? Einer jener Steine, die die Eltern meiner Eltern zum Schutz der Beerensträucher aufeinandergestapelt haben? Der Lichteinfallswinkel auf der Meeresoberfläche? Überhaupt irgend etwas? Vielleicht bloß ein Träumender? ${ }^{9}$

Das wohl interessanteste Beispiel für alle diese Fälle aus der estnischen Literatur bietet die Lyrik des 1941 geborenen Jaan Kaplinski, der als der exophonste Autor Estlands gelten dürfte. Schon in seinem Buch Öölinnud. Öömõtted (1998; dt. Nachtvögel. Nachtgedanken) publizierte Kaplinski neben estnischsprachigen Gedichten eine Reihe von Texten in finnischer und englischer Sprache. Sein Band Sõnad sõnatusse (2005; dt. Worte ins Wortlose) enthält auch russische Gedichte.

Mit seinem zu Ende des Jahres 2010 gemachen Blogeintrag „Goodbye my Estonian“ trennte Kaplinski sich von seiner bisherigen Schaffenssprache und erklärte zugleich, warum er nicht mehr auf Estnisch schreiben könne. Kaplinski entschloss sich, seine Muttersprache gewissermaßen aufzugeben, denn seinem Eindruck nach sind es heute nur noch bestimmte Gruppen von Fachleuten, mit dem damaligen Präsidenten (Toomas Hendrik Ilves) an der Spitze, die den SprecherInnen des Estnischen Worte, Normen und Ausdrucksweisen aufzwingen. Kaplinski wettert gegen die estnische IT-Terminologie, die er nicht für erlernenswert hält, denn sie sei nur scheinbar Estnisch, und er schließt: „Niemand hat das Recht, den Leuten Estlands zu sagen, welche Worte sie benutzen sollten und welche nicht."

Kaplinski schreibt:

Erstens habe ich Gedichte, Essays und Aufsätze in estnischer Sprache seit etwa 50 Jahren, das ist ein halbes Jahrhundert, geschrieben. Genügt das nicht? [...] Ich habe versucht, mein Bestes zu tun, um diese Sprache intelligent und kreativ zu verwenden. Ich habe versucht, sie vor Normativen und Neologismen in Schutz zu nehmen. Jetzt habe ich das Gefühl, dass meine Bemühungen folgenlos geblieben sind. Ich habe meine Schlacht verloren. Es bleibt mir nichts anderes übrig, als mich zu ergeben. Ich will keine estnischen Medien mehr lesen, weder mir unsere Radiosendungen anhören noch unsere Fernsehprogramme angucken. Eine modernisierte Sprache tut mir weh. Ich muss das Schreiben in estnischer Sprache aufgeben und versuchen, fortan auf Werroestnisch, Englisch oder Russisch zu schreiben. ${ }^{10}$

9 Aus dem Estnischen übersetzt von Vahur Aabrams.

10 http://jaankaplinski.blogspot.com/2010/12/goodbye-my-estonian.html. Übersetzt von Vahur Aabrams. 
Zweisprachigkeit in den Literaturen Estlands

Und das tut er auch, von diesem Zeitpunkt an dichtet er auf Englisch, Werroestnisch und Russisch.

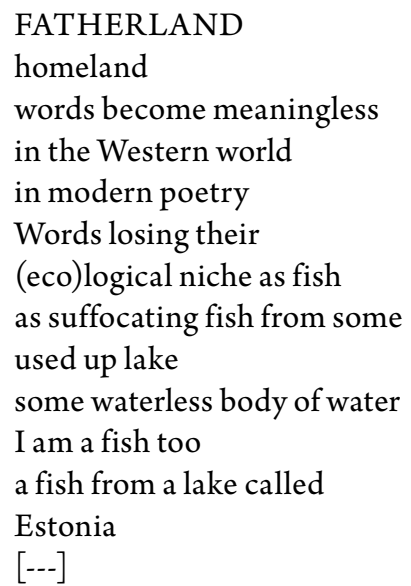

They asked me do I feel myself at least a bit Polish what could I answer them what did I answer them an Estonian non-Catholic non-Protestant a fish from a far-off lake looking upon them through these multicoloured reefs and waves what words have they heard from my mouth grown up in another language in another world [---]

(Kaplinski 2000: 883-886)

Anfang November 2019 erschien das bisher letzte Werk von Jaan Kaplinski, seine Kindheits- und Jugenderinnerungen Latsepõlve suve (Võru, 2019), verfasst auf Werroestnisch - in der Sprache seiner Kindheit und Jugend.

Im Jahre 2014 gab er als Ян Капминский einen Band von Gedichten in russischer Sprache heraus Белые бабочки ночи/Бильля бабочки ночи (Nachwort von Sergei Zawjalow, Kite, 2014; d.h. Weißer Nachtfalter auf Russisch und Altrussisch). Der Band wurde von der russischen Kritik positiv aufgenommen: 
LUKAS

„Nach Rilke ist das der zweite Versuch, dass ein europäischer Dichter von diesem Rang sich dem russischen Vers zuwendet. Im Unterschied zu Rilke dichtet Kaplinski glänzend in russischer Sprache.” (Kanevskij 2014)

Dieser Sprachwechsel erregte in der estnischen Gesellschaft starkes Aufsehen, man sah bzw. suchte darin die Äußerung einer politischen Stellungnahme. Man hat aber auch Kaplinskis Entschluss zu einer grundsätzlichen Mehrsprachigkeit als Zeichen einer zu begrüßenden Interkulturalität interpretiert und dabei auf den Semiotiker Juri Lotman verwiesen, der Mehrsprachigkeit - Berührung und Austausch der Sprachen - als die unentbehrliche Bedingung zur Entwicklung einer Kultur auffasst (vgl. Trunin 2014). So kann der Wechsel der Sprache dem Dichter neue kreative, dichterische Möglichkeiten eröffnen.

Mit diesem Lotmanschen Gedanken wäre es angemessen, diesen kurzen Abriss der Geschichte der estnischen mehrsprachigen und exophonen Literatur zu beschließen. Doch man gestatte mir noch eine Bemerkung. Bei Phänomenen der Mehrsprachigkeit bzw. Exophonie drängt sich immer die Frage auf: welcher Literatur gehört so etwas/jemand eigentlich an? Die deutschbaltischen AutorInnen hielten sich selbst in der Regel für der deutschen Literatur zugehörig. Heute würde man sie dennoch eher der estnischen bzw. lettischen Literatur (oder wenigstens der Literatur Estlands bzw. Lettlands) zurechnen.

Dieselbe Frage hat man sich manchmal auch angesichts der heutigen, estnischrussischen' Literatur gestellt, die sich eigentlich als solche noch erst bewähren muss. Ist sie ein Bestandteil der russischen oder der estnischen Literatur? Ich bin der Meinung, die russischestnische Literatur gehört sehr wohl zur estnischen Literatur. Eine Geschichte der estnischen Literatur sollte folglich auch Kapitel über die deutschbaltische und russischestnische Literatur enthalten.

Bisher hat man das so nicht gemacht, selbst wenn die Idee an sich ja gar nicht so neu ist. So hat der erste Professor für estnische Literatur an der estnischsprachigen Universität Tartu, Gustav Suits, im Jahre 1929 die Aufgabe der Literaturforschung folgendermaßen formuliert: „Baltische Literaturgeschichte in einem weiteren Sinne des Wortes wäre erst dann bearbeitet, wenn der Horizont des Betrachters die literarischen Berührungen und Gegenwirkungen der hiesigen Völker umfasst.“ (Suits 1929: 208). Ihm sekundiert Otto Alexander Webermann, den das Schicksal nach dem Zweiten Weltkrieg nach Göttingen brachte:

Unabhängig davon, wo man die Hauptakzente setzt, kann der Literaturhistoriker in solchen Räumen wie in unserer Heimat, wo mehrere Sprachen nebeneinander existieren und wo in früheren Zeiten die meisten Autoren in zwei oder in mehreren Sprachen geschrieben haben, nicht umhin, einerseits diesen 
Zweisprachigkeit in den Literaturen Estlands

Raum als eine Einheit zu betrachten, andererseits aber den komplexen Charakter der geistigen Erscheinungen zu berücksichtigen, und wenn die bisherigen Möglichkeiten und Methoden nicht ausreichen, so müssen wir nach neuen suchen. (Webermann 1960:27)

\section{Liina Lukas \\ liina.lukas@ut.ee \\ University of Tartu \\ ESTONIA}

\section{Literaturverzeichnis}

Aabrams, V. 2007. „Mehr nurrige Gesicht“. Vier Gedichte in estnischem Halbdeutsch. Aus einer karnevalesken Umbruchszeit. Magisterarbeit (Manuskript). Universität Tartu, 2007.

Ariste, P. 1981. Keele kreolisatsioon. - Ariste, P. Keelekontaktid. Eesti keele kontakte teiste keeltega. Tallinn: Valgus, 70-75.

Arndt, S., Naguschewski, D., Stockhammer, R., Hrsg. 2007. Exophonie. AndersSprachigkeit (in) der Literatur. Berlin: Kulturverlag Kadmos.

Belobrovtseva, I. 2018. The Bilingual Writer: Two Estonian-Russian Cases and One Russian-Estonian Case. - Methis, 21/22, 8-25. https://doi.org/10.7592/methis. v17i21/22.14582

Brockmann, R. 2000. Teosed $=$ PEINEPOY BPOKMANNOY $\Pi$ OIHMATA $=$ Reiner $i$ Brockmanni opera = Reiner Brockmann's Werke. Priidel, E., Hrsg. Tartu: Ilmamaa.

Eisenreich, H. 1967. Vorwort. - I. Ivask, Gespiegelte Erde: Gedichte 1953-1963. New York: Ungar, 1967, 5-7.

Fleming, P. 1636. Lieffländische Schneegräfinn, auff H. Andres Rüttings, Und Jungfr. Annen von Holten Hochzeit. Revall, 1636 [Manuskript, unpaginiert].

Hasselblatt, C. 1997. Der Gedanke der Kulturautonomie und seine gesetzliche und organisatorische Verwirklichung. - B. Meissner, D. A. Loeber, C. Hasselblatt, Hrsg., Die deutsche Volksgruppe in Estland während der Zwischenkriegszeit und aktuelle Fragen des deutsch-estnischen Verhältnisses. Hamburg: Bibliotheca Baltica, $37-46$.

Johansen, P., Mühlen, H. v. z. 1973. Deutsch und undeutsch im mittelalterlichen und frühneuzeitlichen Reval. [Ostmitteleuropa in Vergangenheit und Gegenwart 15]. Köln/ Wien: Böhlau.

Kalda, M. 2000. Jacob Johann Malm ja makarooniline luule. - Kalda, M. Mis mees ta on? [Collegium litterarum 12]. Tallinn: Eesti Teaduste Akadeemia Underi ja Tuglase Kirjanduskeskus, 82-149.

Kanevskij, G., Oborin, L., Sungatov, N. 2014. O knige Ya. Kaplinskoge "Belye babočki

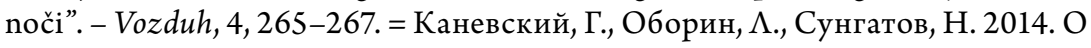
книге Я. Капминского «Бемые бабочки ночи». - Возд $y x, 4,265-267$. 
LUKAS

Kaplinski, J. 2000. Kirjutatud. Valitud luuletused. Tallinn: Varrak.

Kitzberg, A. 2002. Veli Henn. - Kitzberg, A. Külajutud. Tallinn: Eesti Raamat, 262-343.

Kivisilla, V. 2018. Kuni armastus peale tuleb. Tallinn: Hunt.

Kotjuh, I. 2007. Teises keeles: essee, luuletused ja esseemid 2000-2007. Tallinn: Tuum.

Lehiste, I. 1965. A poem in Halbdeutsch and some questions concerning substratum. World, 21, 33-69. https://doi.org/10.1080/00437956.1965.11435418

Malm, J. J. 1861. Die Oberpalsche Freundschaft. Reval.

Masing, O. 1923. Baltisches Deutsch. - Zeitschrift für Deutschkunde, 37 (2), 83-89.

Rangøy, Ø. 2019. Sisikond. Tallinn: Vihmakass ja kakerdaja.

Stein, P. 2006. Schriftkultur. Eine Geschichte des Schreibens und Lesens. Darmstadt: Wissenschaftliche Buchgesellschaft.

Suits, G. 1929. Balti kirjandusloo katse. - Eesti Kirjandus, 5, 193-209.

Trunin, M. 2014. Mõned mõtted Jaan Kaplinski venekeelse luuletaamatu kohta. - Sirp. Eesti Kultuurileht, https://www.sirp.ee/s1-artiklid/c7-kirjandus/2014-07-09-22-03-08/, 09.07.2014.

Undusk, J. 1992. Saksa-eesti kirjandussuhete tüpoloogia. - Keel ja Kirjandus, 10, 583594; 11, 645-656; 12, 709-725.

Undusk, J. 1999. Adressat und Sprache im deutschbaltischen Kulturraum. - U. Obst, G. Ressel, Hrsg., Balten - Slaven - Deutsche: Aspekte und Perspektiven kultureller Kontakte: Festschrift für Friedrich Scholz zum 70. Geburtstag. Münster-Hamburg-London: LIT, 347-361.

Verschik, A. 2005. Russian-Estonian Language Contacts, Linguistic Creativity, and Convergence: New Rules in the Making. - Multilingua, 24 (4), 413-429. https:// doi.org/10.1515/mult.2005.24.4.413

Verschik, A., Bone, E. 2018. Understanding Linguistic Features of Estonian-Latvian Bilingual Speech. - P. Romanowski, M. Jedynak, Hrsg., Current Research in Bilingualism and Bilingual Education. Springer, 79-102. https://doi.org/10.1007/978-3-319-923963_5

Webermann, O. A. 1960. Deutschbaltische und estnische Literatur. - Baltische Hefte, 7 (1), 17-28. 